\title{
PRECISE FINITE SPEED WITH BARE HANDS*
}

\author{
JEFFREY RAUCH ${ }^{\dagger}$
}

Dedication. I am pleased to dedicate this article to my friend and colleague Joel Smoller. Joel advised and recruited me at the beginning of my career and is largely responsible for the warm welcome I received at the University of Michigan. His seminar, teaching, and research have been an inspiration to me for more than three decades.

Abstract. A precise finite speed result is proved for general hyperbolic systems using neither Hamilton-Jacobi Theory as in [9] nor the theorem of Marchaud [12] as in Leray [11]. The proof is shorter than both [11] and [9], and more general than the first. It does not give the additional information about spacelike deformations proved in [9].

Key words.

AMS subject classifications.

1. Introduction. For a system of partial differential operators whose principal part is hyperbolic, the propagation cone at a point is the cone associated to the constant coefficient homogeneous operator obtained by freezing coefficients and dropping lower order terms. An influence curve is a curve $(t, \gamma(t))$ whose tangent at almost all times belongs to the local propagation cone. By precise finite speed we mean the fact that the values of Cauchy data in a set $K$ influence the solution only at points which are accessible by influence curves as stated, for example, in [3].

At the heart of the proofs here and in [10] and [11] is a local result for operators whose coefficients are nearly constant. In [10] and [11] such results are proved by energy methods. Here we use deformation of space like hypersurfaces (see Lemma 8). Precise finite speed is proved with the single important hypothesis of local uniqueness in the Cauchy problem at space like hypersurfaces. The passage from local to global is handled differently than earlier proofs and with bare hands. For strictly hyperbolic equations, Leray [11] uses a nontrivial geometric result of Marchaud [12]. Joly, Métivier and Rauch [9] use Hamilton-Jacobi Theory. Lax's sketch of proof in [10] for the symmetric hyperbolic case glosses the point. Our passage from the the local Lemma 8 to global is a compactness-connectedness argument. For the symmetric case, we give an alternate proof in $\S 3$. It is the shortest proof we know. See [6] for a similar strategy in the scalar second order case.

1.1. Timelike and propagation cones. Denote

$$
(t, x)=\left(t, x_{1}, \ldots, x_{d}\right)=y=\left(y_{0}, y_{1}, \ldots, y_{d}\right) \in \mathbb{R}^{1+d},
$$

with dual variable

$$
(\tau, \xi)=\left(\tau, \xi_{1}, \ldots, \xi_{d}\right)=\eta=\left(\eta_{0}, \eta_{1}, \ldots, \eta_{d}\right) .
$$

Definition 1. A homogeneous polynomial $p(\tau, \xi)$ is hyperbolic with timelike variable $t$ when $p(1,0, \ldots, 0) \neq 0$ and for all $\xi \in \mathbb{R}^{d}$, the roots $\tau$ of $p(\tau, \xi)=0$ are all

\footnotetext{
${ }^{*}$ Received June 28, 2005; accepted for publication November 25, 2005.

$\dagger$ Department of Mathematics, University of Michigan, 530 Church Street, Ann Arbor, MI 481091043, USA (rauch@umich.edu). Research partially supported by the National Science Foundation under grant NSF DMS 0405899.
} 
real. For such a polynomial and $\xi \in \mathbb{R}^{d}$, define

$$
\tau_{\max }(\xi):=\max \{\tau: p(\tau, \xi)=0\}
$$

The open time like cone is

$$
\mathcal{T}:=\left\{(\tau . \xi) \in \mathbb{R}^{1+d} \backslash 0: \tau>\tau_{\max }(\xi)\right\} .
$$

The timelike cone is the connected component in $\left\{(\tau, \xi) \in \mathbb{R}^{1+d} \backslash 0: p(\tau, \xi) \neq 0\right\}$ which contains the covector $(1,0, \ldots, 0)$. The time like cone and the function $\tau_{\max }$ are convex (see [5]).

Definition 2. The propagation cone, $\Gamma^{+}$is the closed dual cone to $\mathcal{T}$,

$$
\Gamma^{+}:=\{(t, x) ; \forall(\tau, \xi) \in \mathcal{T}, \tau t+\xi . x \geq 0\} .
$$

The section at $t=1$ is denoted

$$
\Gamma_{1}^{+}:=\Gamma^{+} \cap\{t=1\}
$$

For constant coefficient hyperbolic partial differential operators, $\Gamma^{+}$is equal to the convex hull of the support of the forward fundamental solution (see [5]).

The definition implies that $\mathcal{T}$ is the open dual cone to $\Gamma^{+}$,

$$
\mathcal{T}=\left\{(\tau, \xi): \forall(t, x) \in \Gamma^{+}: \tau t+\xi \cdot x>0\right\} .
$$

Comparing this with (2) yields

$$
\tau_{\max }(\xi)=\max _{x \in \Gamma_{1}^{+}}\{-\xi . x\} .
$$

The hyperplanes $\{\tau t+\xi . x=0\}$ which meet $\Gamma^{ \pm}$only at the origin in $\mathbb{R}_{t, x}^{1+d}$ are those with conormals in $\mathcal{T}$. These are the hyperplanes that separate $\Gamma^{+} \backslash 0$ from $\Gamma^{-} \backslash 0$ $\left(\Gamma^{-}:=-\Gamma^{+}\right)$.

\subsection{Hyperbolic systems.}

Definition 3. A system of partial differential operators

$$
L\left(y, \partial_{y}\right):=\partial_{t}+\sum_{j=1}^{d} A_{j}(y) \partial_{j}+B(y)
$$

is hyperbolic on $[0, T] \times \mathbb{R}^{d}$ when

$$
\forall \alpha \sup _{y \in[0, T] \times \mathbb{R}^{d}}\left|\partial_{y}^{\alpha}\left\{A_{j}(y), B(y)\right\}\right|<\infty,
$$

and, for all $y \in[0, T] \times \mathbb{R}^{d}$,

$$
p(y, \tau, \xi):=\operatorname{det}\left(\tau I+\sum_{j} A_{j}(y) \xi_{j}\right)
$$


is a hyperbolic polynomial in $(\tau, \xi)$. The system is symmetric hyperbolic (see [4], [10]) if in addition to (3), the matrices $A_{j}$ are hermitian symmetric.

For symmetric hyperbolic systems, $p(y, \tau, \xi)$ is automatically hyperbolic.

For hyperbolic systems, $\tau_{\max }(y, \xi), \Gamma^{+}(y)$, and $\mathcal{T}(y)$ depend on $y$. The smoothness in (3), implies by a Theorem of Bronstein (see [1], [15]) that $\tau_{\max }$ is uniformly lipschitzean on compacts. The propagation and time like cones inherit this regularity.

The fact that the $A_{j}$ are uniformly bounded implies that the $\Gamma_{1}^{+}(y)$ are bounded uniformly in $y$. This implies, for example from Theorem 1, a global bound on the propagation speeds.

1.3. Influence curves. Definition 4. A lipschitz curve $[a, b] \ni t \mapsto(t, \gamma(t))$ is an influence curve when for almost all $t,\left(1, \gamma^{\prime}(t)\right) \in \Gamma^{+}(t, \gamma(t))$. The curve $(-t, \gamma(t))$ a backward influence curve when $\left(-1, \gamma^{\prime}\right) \in-\Gamma^{+}(-t, \gamma(t))$ for almost all $t$.

The uniform boundedness of the sets $\Gamma_{1}^{+}$implies that influence curves are uniformly lipschitzean. Peano's existence proof combining Euler's scheme and Ascoli's Theorem, implies that influence curves exist with arbitrary initial values.

The convexity of the sets $\Gamma_{1}^{+}$implies that uniform limits of influence curves are influence curves. Indeed, if $y^{n}(t)=\left(t, \gamma_{n}(t)\right)$ is such a uniformly convergent sequence, then $\gamma_{n}^{\prime} \in \Gamma_{1}^{+}\left(t, \gamma_{n}(t)\right)$. This uniform bound allows us to pass to a subsequence for which

$$
\gamma_{n}^{\prime} \quad \rightarrow \quad f(t)
$$

weak star in $L^{\infty}([a, b])$. Since the $\Gamma_{1}^{+}$are convex one has $f(t) \in \Gamma_{1}^{+}(t, \gamma(t))$ for almost all $t$.

On the other hand, $g_{n}^{\prime}$ converges to $g^{\prime}$ in the sense of distributions. Therefore $g^{\prime}=f$ and therefore $(t, \gamma(t))$ is an influence curve.

Ascoli's Theorem implies that from any sequence of influence curves defined on $[a, b]$ whose initial points lie in a bounded set, one can extract a uniformly convergent subsequence.

Following Leray [11], define emissions as follows.

Definition 5. If $K \subset[0, T] \times \mathbb{R}^{d}$ is a closed set, the forward emission of $K$ denoted $\mathcal{E}^{+}(K)$ is the union of forward influence curves beginning in $K$. The backward emission is denoted $\mathcal{E}^{-}$.

The emissions are closed subsets of $[0, T] \times \mathbb{R}^{d}$.

1.4. Main theorem. Definition 6. A smooth embedded hypersurface, $S \subset$ $[0, T] \times \mathbb{R}^{d}$, is space like at $p \in[0, T] \times \mathbb{R}^{d}$ if it has a conormal in $\mathcal{T}(p)$.

It follows from continuity with respect to $y$ of conormals and of $\mathcal{T}(y)$, that $S$ is space like on a neighborhood of $p$.

Definition 7. The hyperbolic operator $L$ has local uniqueness in the Cauchy problem at space like hypersurfaces if whenever $S$ is an open piece of hypersurface lying in $[0, T] \times \mathbb{R}^{d}$ which is space like at $p$ and $u \in C^{\infty}$ satisfies Lu $=0$ on a neighborhood of $p$ and $\left.u\right|_{S}$ vanishes on a neighborhood of $p$, then $u$ vanishes on a neighborhood of $p$ in $[0, T] \times \mathbb{R}^{d}$.

This definition permits the possibility that $S$ is a piece of $\{t=0\}$. 
EXAMPLES. 1. Symmetric hyperbolic systems. 2. Strictly hyperbolic systems. 3. Generalizing the latter are systems with characteristic variety smooth and of constant multiplicity with diagonalisable principal symbol. 4. Systems with analytic coefficients and hyperbolic principal part. The local uniuqeness follows from Hölmgren's Theorem. 5. Systems with hyperbolic principal part and coefficients in the Gevrey class $G^{s}\left([0, T] \times \mathbb{R}^{d}\right)$ with $1<s<N / N-1$. Local uniqueness follows from the existence theorem of Bronshtein [2] by a Hölmgren style duality. Bronsthein's results are recalled in $\S 2.2$ of [7] and have a new proof in [14].

For the first three there are good $L^{2}$ estimates, while for the last two the known estimates are very weak. One of the good things about the proofs in [9] and in this paper, is that they do not require strong estimates. Both this paper and [9] include shorter and independent proofs in the symmetric hyperbolic case.

The main theorem of this paper is the following.

TheOrem 1. If $L$ is a hyperbolic system which has local uniqueness in the Cauchy problem at spacellike hypersurfaces in $[0, T] \times \mathbb{R}^{d}$ and $u \in C^{\infty}\left([0, T] \times \mathbb{R}^{d}\right)$ satisfies $L u=0$ then the support of $u$ is contained in $\mathcal{E}^{+}\left(\operatorname{supp}\left(\left.u\right|_{t=0}\right)\right)$.

REMARK. For problems for which there is a good existence theorem for the Cauchy problem, for example the symmetric or constant multiplicity cases, one gets the same bound on support for distribution initial data by an approximation argument.

The main theorem accurately describe the domain of influence of the Cauchy data. For a discussion of the adjacent notions of domain of dependence, and relations with Hamilton-Joacobi theory see [9].

2. Fattened propagation cones. The fattening gives one a little wiggle room, as in both [11] and [10]. It also regularizes the boundary of emissions as in [9]. Using (1) and (2), we fatten $\Gamma$ by shrinking $\mathcal{T}$.

Definition 8. For $\varepsilon>0$, define the shrunken time like cone,

$$
\mathcal{T}^{\varepsilon}(y):=\left\{(\tau, \xi) \in \mathbb{R}^{1+d}: \tau>\tau_{\max }(y, \xi)+\varepsilon|\xi|\right\} .
$$

Define the fattened propagation cone, $\Gamma^{+, \varepsilon}(y)$, to be the closed dual cone. Denote by $\mathcal{E}_{\varepsilon}^{ \pm}$the emissions defined with the $\Gamma^{ \pm, \varepsilon}$.

The fattened cones, $\Gamma^{+, \varepsilon}(y)$ are strictly convex, increasing in $\varepsilon$ and contain $\Gamma^{+, \varepsilon / 2}(y) \backslash 0$ in their interior. In addition, $\cap_{0<\varepsilon<1} \Gamma^{+, \varepsilon}(y)=\Gamma^{+}(y)$.

Lemma 2. To prove Theorem 1, it suffices to show that if $\varepsilon>0, \underline{y} \in[0, T] \times \mathbb{R}^{d}$, and, $\mathcal{E}_{\varepsilon}^{-}(\underline{y})$ does not meet $\operatorname{supp}\left(\left.u\right|_{t=0}\right)$, then u vanishes on $\mathcal{E}_{\varepsilon}^{-}(\underline{y})$.

Proof. To prove Theorem 1 one must show that

$$
[0, T] \times \mathbb{R}^{d} \ni y \notin \mathcal{E}^{+}(\operatorname{supp}(u(0, \cdot)) \quad \Longrightarrow \quad y \notin \operatorname{supp}(u) .
$$

If $y \notin \mathcal{E}^{+}\left(\operatorname{supp}(u(0, \cdot))\right.$, then points $\underline{y}$ on a neighborhood of $y$ in $[0, T] \times \mathbb{R}^{d}$ are also not in $\mathcal{E}^{+}(\operatorname{supp}(u(0, \cdot))$. Therefore it suffices to show that

$$
[0, T] \times \mathbb{R}^{d} \ni \underline{y} \notin \mathcal{E}^{+}(\operatorname{supp}(u(0, \cdot)) \quad \Longrightarrow \quad u(\underline{y})=0 .
$$

From the definitions,

$$
\underline{y} \notin \mathcal{E}^{+}\left(\operatorname{supp}(u(0, \cdot)) \quad \Longleftrightarrow \quad \mathcal{E}^{-}(\underline{y}) \cap \operatorname{supp}(u(0, \cdot))=\phi .\right.
$$


The compact sets $\mathcal{E}_{\varepsilon}^{-}(\underline{y})$ decrease as $\varepsilon$ decreases, and,

$$
\cap_{0<\varepsilon<1} \mathcal{E}_{\varepsilon}^{-}(\underline{y})=\mathcal{E}^{-}(\underline{y}) .
$$

Therefore, for $\varepsilon$ small, $\mathcal{E}_{\varepsilon}^{-}(\underline{y})$ does not meet $\operatorname{supp}\left(\left.u\right|_{t=0}\right)$.

Therefore, to prove Theorem 1, it suffices to show that if $\mathcal{E}_{\varepsilon}^{-}(\underline{y})$ does not meet $\operatorname{supp}(u(0, \cdot)$ then $u(\underline{y})=0$. This is equivalent to the statement of the lemma.

3. A short symmetric hyperbolic proof . While $\Gamma^{+}(y)$ can be a lower dimensional cone, $\Gamma^{+, \varepsilon}(y)$ has nonempty interior. The next lemmas are used to give a simple proof for the symmetric case. They are not used in the proof of the general case of Theorem 1, so readers only interested in the general case can omit this section.

The next lemma is an accessibility theorem, in the sense of control theory. so that

Lemma 3. If $\underline{y}=(\underline{t}, \underline{x}) \in\left[0, T\left[\times \mathbb{R}^{d}\right.\right.$ and $\left.\varepsilon \in\right] 0,1[$, then there is a $0<\delta \leq T-\underline{t}$

$$
\mathcal{E}_{\varepsilon}^{+}(\underline{y}) \supset\left\{\underline{y}+\Gamma^{+, \varepsilon / 2}(\underline{y})\right\} \cap\{\underline{t} \leq t \leq t+\delta\} .
$$

$\delta$ can be chosen uniformly for $y$ in compact subsets of $\left[0, T\left[\times \mathbb{R}^{d}\right.\right.$. An analogous result holds for backward emissions.

Proof. Continuity of $\Gamma^{+, \varepsilon}(y)$ with respect to $y$ implies that there is a $\delta_{0}$ so that

$$
|y-\underline{y}|<\delta_{0} \quad \Longrightarrow \quad \Gamma^{+, \varepsilon}(y) \supset \Gamma^{+, \varepsilon / 2}(\underline{y}) .
$$

Therefore, a curve $(t, \gamma(t))$ with $\left(1, \gamma^{\prime}\right) \in \Gamma^{+, \varepsilon / 2}(\underline{y})$ is an influence curve so long as it stays in $\left\{|y-\underline{y}|<\delta_{0}\right\}$. Choose $0<\delta \leq \delta_{0}$ so that this holds for $t \in[\underline{t}, \underline{t}+\delta]$ on influence curves starting in $\{|y-\underline{y}|<\delta\}$. This completes the proof for $y$ fixed.

That the constants can be chosen uniformly follows from the fact that $\Gamma^{+}(y)$ is uniformly continuous on compact sets.

Lemma 4. For any $q$ and $\varepsilon>0$, the set $\mathcal{E}_{\varepsilon}^{-}(q)$ has lipschitz boundary. The boundary has a tangent plane at almost all points. At such points, the conormals belong to $\mathcal{T} \cup-\mathcal{T}$.

Proof. Suppose that $\underline{y} \neq q$ belongs to the boundary of $\mathcal{E}_{\varepsilon}^{-}(q)$. Then for $t$ close to and greater than $\underline{t}, \underline{y}+\Gamma^{+, \varepsilon / 4}(\underline{y})$ belongs to the complement of $\mathcal{E}_{\varepsilon}^{-}(q)$. To prove this note that if there were points

$$
z=(t, x) \in \underline{y}+\Gamma^{+, \varepsilon / 4}(\underline{y}) \cap \mathcal{E}_{\varepsilon}^{-}(q) \quad \text { with } \quad \underline{t}<t<\underline{t}+\delta,
$$

as in the figure below, then $\mathcal{E}_{\varepsilon}^{-}(z) \subset \mathcal{E}_{\varepsilon}^{-}(q)$. Lemma 3 implies that $\mathcal{E}_{\varepsilon}^{-}(z)$ contains a neighborhood of $\underline{y}$. contradicting the fact that $\underline{y}$ is a boundary point.

On the other hand, since $\underline{y}$ belongs the emission, $\mathcal{E}_{\varepsilon}^{-}(\underline{y})$ belongs the emission. Lemma 3 implies that for $\underline{t}>t>t-\delta$, the emission from $\underline{y}$ contains $\underline{y}-\Gamma_{1}^{+, \varepsilon / 2}(\underline{y})$. The interior of that set is thus a subset of the interior of the emission. Thus the boundary of the emmision near $\underline{y}$ is sandwiched between $\underline{y}+\Gamma^{+, \varepsilon / 4}(\underline{y})$ and $\underline{y}-\Gamma^{+, \varepsilon / 4}(\underline{y})$ as in the figure below. 


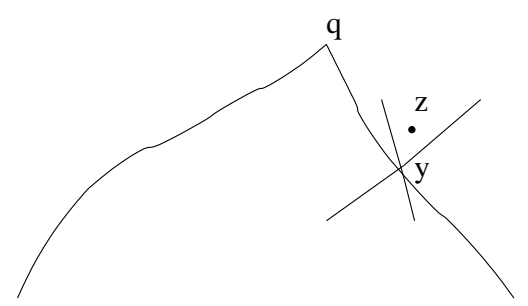

FIG. 1

At $\underline{y}=q$, it is also true that the boundary of $\mathcal{E}_{\varepsilon}(q)$ is sandwiched between $\underline{y}+$ $\Gamma^{+, \varepsilon / 4}(\underline{y})$ and $\underline{y}-\Gamma^{+, \varepsilon / 4}(\underline{y})$.

This shows that at all points, the boundary satisfies a two sided cone condition with cones $\pm \Gamma^{+, \varepsilon / 2}(\underline{y})$ which are lipschitzean in their dependence on $\underline{y}$. This proves the desired lipschitz regularity of the boundary. The differentiability then follows from Rademacher's Theorem asserting the almost everywhere differentiability of lipschitz functions.

At points, $\underline{y}$, of differentiability, the tangent plane locally separates the cones $\underline{y} \pm \Gamma^{+, \varepsilon / 4}(\underline{y})$. This implies that the plane separates the smaller cones $\underline{y} \pm \Gamma^{+}(\underline{y})$. In $\S 1.1$ it was noted that such separating planes are exactly those which have conormals in $\mathcal{T}(\underline{y}) \cup-\mathcal{T}(\underline{y})$.

Proof of Theorem 1 in the symmetric case. We verify the criterion of Lemma 2. For $0<t<\underline{t}$ define

$$
\left.\Omega_{t}:=\mathcal{E}_{\varepsilon}^{-}(\underline{y}) \cap[0, t] \times \mathbb{R}^{d}, \quad \mathcal{B}_{t}:=\partial \mathcal{E}_{\varepsilon}^{-}(\underline{y}) \cap\right] 0, t\left[\times \mathbb{R}^{d} .\right.
$$

The set $\mathcal{B}_{t}$ is the lateral boundary of $\Omega_{t}$.

The energy conservation law in the symmetric case is

$$
2 \operatorname{Re}(L u, u)=\partial_{t}(u, u)+\sum_{j} \partial_{j}\left(A_{j} u, u\right)+(\mathbf{Z} u, u)
$$

where

$$
\mathbf{Z}(y):=B+B^{*}-\sum_{j} \partial_{j} A_{j},
$$

Define

$$
\phi(s):=\int_{\mathcal{E}_{\varepsilon}^{-}(\underline{y}) \cap\{t=s\}}|u(s, x)|^{2} d x .
$$

For smooth solutions of $L u=0$ and $0<t<\underline{t}$, one has

$$
0=\int_{\Omega_{t}} \partial_{t}(u, u)+\sum_{j} \partial_{j}\left(A_{j} u, u\right)+(\mathbf{Z} u, u) d x d t,
$$

Integrating by parts yields

$$
\phi(t)-\phi(0)+\int_{\mathcal{B}_{t}}\left(\sum_{\mu=0}^{d} \nu_{\mu} A_{\mu} u, u\right) d \sigma+\int_{\Omega_{t}}(\mathbf{Z} u, u) d x d t=0,
$$


where $\nu$ is the unit outward normal to $\mathcal{B}$ and $d \sigma$ is the element of $d$ dimensional surface area in the boundary $\mathcal{B}$. The conormal $\nu$ is almost everywhere well defined with respect to surface area. From Lemma $4, \nu$ belongs to $\mathcal{T} \cup-\mathcal{T}$. Since $\mathcal{E}_{\varepsilon}^{-}(\underline{y})$ is a backward emission, the outward conormals belong to $\mathcal{T}$. Therefore the matrix $\sum_{\mu} \nu_{\mu} A_{\mu}$ is strictly positive. Using this in (4) yields

$$
\phi(t) \leq \phi(0)+\|Z\|_{L^{\infty}\left([0, T] \times \mathbb{R}^{d}\right)} \int_{0}^{t} \phi(s) d s .
$$

Since $\mathcal{E}^{-}$does not meet the support of $\left.u\right|_{t=0}, \phi(0)=0$. Gronwall's Lemma implies that $\phi(t)=0$ for $0 \leq t \leq \underline{t}$. This shows that $u$ vanishes in $\mathcal{E}_{\varepsilon}^{-}(\underline{y})$ so completes the proof of the Theorem 1 in the symmetric case.

4. The method of space like deformations. The proof in the general case propagates zeroes of the solution using local uniqueness in the Cauchy problem and the method of space like deformations. This method uses the geometric idea behind F. John's Global Hölmgren Theorem (see [8], [13]). In John's Theorem, local uniqueness is implied by Hölmgren's Theorem. In our application, it is local uniqueness at space like hypersurfaces that is used.

Proposition 5 below asserts that solutions of $L u=0$ vanish on domains swept out by space like surfaces with their feet in the set where the Cauchy data of $u$ vanish. The two simple examples of $\partial_{t}^{2}-\partial_{x}^{2}$ and $\partial_{t}+\partial_{x}$ both in dimension $d=1$ with an initial data vanishing in ] - 1, 1 [ give the essential idea of the method and are sketched in Figure 2.
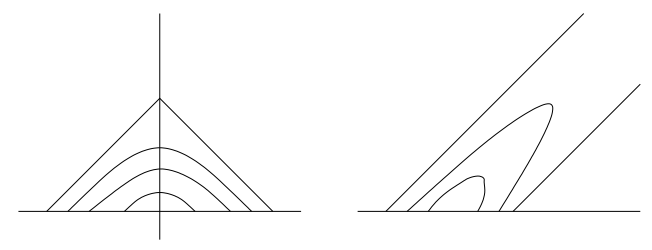

FIG. 2

In the next result the deformation is by the level sets $\{F=c\}$ with $c$ increasing from 0 to 1 .

Proposition 5. Suppose that $L$ has local uniqueness at space like hypersurfaces, that $\mathcal{O}$ is a relatively open subset of $[0, T] \times \mathbb{R}^{d}$ and $F \in C^{1}(\mathcal{O})$ has the following properties.

i. $F>0$ on $\mathcal{O} \cap\{t>0\}$.

ii. $F^{-1}([0,1]) \subset \subset \mathcal{O}$.

iii. For all $y \in F^{-1}([0,1]), d F(y) \in \mathcal{T}(y)$.

Then, if $u \in C^{\infty}\left([0, T] \times \mathbb{R}^{d}\right)$ satisfies Lu $=0$ on $\mathcal{O}$, and, $\left.u\right|_{t=0}$ vanishes on a neighborhood of $F^{-1}([0,1]) \cap\{t=0\}$, then $u=0$ on $F^{-1}([0,1])$.

5. Proof of Theorem 1. The first step is an observation about cones.

Lemma 6. Suppose that $\Gamma \subset\{t \geq 0\}$ is a convex cone, $\Gamma_{1}:=\Gamma \cap\{t=1\}$ is compact, and, $\underline{y}=(1, \underline{x}) \in \operatorname{Interior}(\Gamma)$. Then for each point $(0, x) \in\{t=0\}$ the line

$$
t \mapsto(0, x)+t \underline{y}=(t, x+t \underline{x}),
$$


meets $\Gamma$ for $t \geq g(x)$ defining a convex function $g$ positive homogeneous of degree one. In addition, $\{x: g(x) \leq 1\}$ is compact (see Figure 3).

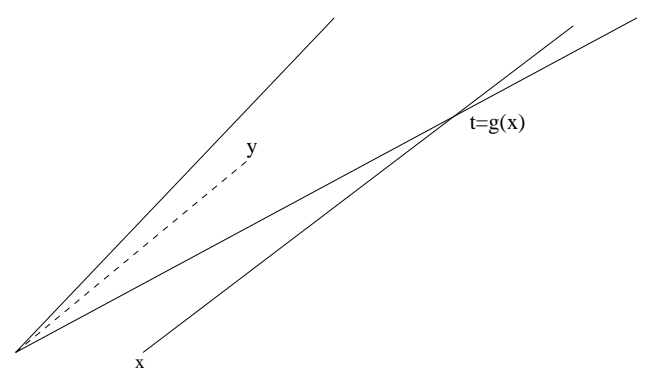

FIG. 3

Proof. Choose a $\rho>0$ so that

$$
\{t=1\} \times\{|x-\underline{x}|<\rho\} \subset \Gamma_{1} .
$$

Then $(t, x) \in \Gamma$ whenever $|x-\underline{x}|<\rho t$. The line meets $\{|x-\underline{x}|<\rho t\}$ in a half line so meets the convex $\Gamma$ in an interval containing a half line. Since $\Gamma$ is contained in $t \geq 0$ it meets $\Gamma$ in exactly a half line.

The shear

$$
S(t, x):=(\tilde{t}, \tilde{x}):=(t, x-\underline{x} t)
$$

with inverse

$$
(t, x)=S^{-1}(\tilde{t}, \tilde{x})=(\tilde{t}, \tilde{x}+\underline{x} t),
$$

maps $\tilde{\Gamma}:=\{\tilde{t} \geq g(\tilde{x})\}$ to $\Gamma=\{t \geq g(x-\underline{x} t)\}$. Since the shear is invertible and linear the convexity of $\tilde{\Gamma}$ and compactness of $\{g(\tilde{x}) \leq 1\}$ follow from the convexity of $\Gamma$ and the compactness of $\Gamma_{1}$.

The next lemma concerns smoothing and fattening of functions like $g$ from the preceding lemma. It concerns the convex cone $\tilde{\Gamma}=\{\tilde{t} \geq g(\tilde{x})\}$, a thinner convex cone $\tilde{t} \geq g(\tilde{x})+\varepsilon|\tilde{x}|$, and a smoothing of the latter. The smoothing is performed by convolution with $j^{\varepsilon}(\tilde{x}):=\varepsilon^{-d} j(\tilde{x} / \varepsilon)$ where $0 \leq j \in C_{0}^{\infty}\left(\mathbb{R}^{d}\right)$, with $\int j d \tilde{x}=1$.

Lemma 7. Suppose that $g: \mathbb{R}^{d} \rightarrow \mathbb{R}$ is a nonnegative convex function homogeneous of degree one so that $\{g(\tilde{x}) \leq 1\}$ is compact. Denote by $\tilde{\Gamma}$ the cone $\{(\tilde{t}, \tilde{x}): \tilde{t} \geq g(\tilde{x})\}, \tilde{\mathcal{T}} \subset \mathbb{R}_{\tilde{\tau}, \tilde{\xi}}^{1+d} \backslash 0$ the open dual cone, and $\tilde{\mathcal{T}}_{1}:=\tilde{\mathcal{T}} \cap\{\tilde{\mathcal{\tau}}=1\}$. Then,

$$
g^{\varepsilon}:=j^{\varepsilon} *(g+\varepsilon|\tilde{x}|)=\int(g(\tilde{x}-\varepsilon \tilde{z})+\varepsilon|\tilde{x}-\varepsilon \tilde{z}|) j(\tilde{z}) d \tilde{z} \in C^{\infty}\left(\mathbb{R}^{d}\right),
$$

is strictly greater than $g$. In addition, $\left(1,-\nabla_{\tilde{x}} g^{\varepsilon}(\tilde{x})\right) \in \tilde{\mathcal{T}}_{1}$.

Proof. The convexity of $g$ and $|\tilde{x}|$ imply that $g * j^{\varepsilon} \geq g$ and $|\tilde{x}| * j^{\varepsilon}>|\tilde{x}|$, so $g^{\varepsilon}>f$.

The vector $\left.\left(1,-\nabla_{\tilde{x}} g^{\varepsilon}\right)\right)$ is an average of covectors vectors $\left(1,-\nabla_{\tilde{x}} g\right)$. From the definition of $\tilde{\mathcal{T}}$ as the dual cone, it follows that each of the latter covectors belongs to the convex set $\tilde{\mathcal{T}}_{1}$. The lemma follows. 
The next result proves uniqueness when the propagation cones are contained in a fixed cone $\Gamma$. We will use it locally where the uniform bound on the cones comes from their continuous dependence on $y$. Analogous results were given in [11] and [10] in the strictly and symmetric hyperbolic cases respectively.

Lemma 8. Suppose that $\Gamma \subset\{t \geq 0\}$ is a convex cone with nonempty interior and $\Gamma_{1}$ compact. For $\left.\left.\underline{z}=(\underline{t}, \underline{x}) \in\right] 0, T\right] \times \mathbb{R}^{d}$ and $\left.\left.\delta \in\right] 0, \underline{t}\right]$, define (see Figure 4),

$$
\Omega:=\Omega(\underline{z}, \delta):=\{\{\underline{z}-\Gamma\} \cap\{\underline{t}-\delta \leq t \leq \underline{t}\}\}, \quad \omega:=\Omega \cap\{t=\underline{t}-\delta\} .
$$

If for all $y \in \Omega, \Gamma^{+}(y) \subset \Gamma$, then

$$
u \in C^{\infty}(\Omega), \quad L u=0 \text { in } \Omega, \text { and }\left.u\right|_{\omega}=0,
$$

imply $u=0$ on $\Omega$.

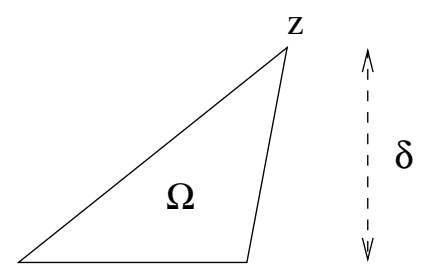

$\omega$

FIG. 4

Proof. Translating time coordinates it suffices to consider the case $\underline{t}-\delta=0$. Translating $x$ coordinates we may suppose that $(0,0) \in \operatorname{Interior}(\omega)$. Therefore, $(0,0)-$ $\underline{z} \in \operatorname{Interior}(-\Gamma)$, equivalently $\underline{z} \in \operatorname{Interior}(\Gamma)$. Denote $\underline{z}=(\underline{t}, \underline{x})$. Define $g$ as in Lemma 6 so the boundary of $\Gamma$ has equation $t=g(x-t \underline{x})$.

Since every boundary point of $\Gamma$ is a limit of interior points, it follows that every boundary point of $\Omega$ is a limit of interior points. Therefore it suffices to show that $u(y)=0$ at every $y \in \operatorname{Interior}(\Omega)$.

This is proved by spacelike deformation. At such a $y, t-g(x-\underline{x} t)<0$. Smooth $g$ as in the preceding lemma. Choose $\varepsilon>0$ so small that $y \in\left\{t-g^{\underline{\varepsilon}}(x-\underline{x} t)<0\right\}$.

We construct $F$ so that Proposition 5 applies, $\{F=1\}=\left\{t=g^{\underline{\varepsilon}}(x-\underline{x} t)\right\}$, and, the level sets of $F$ are translates by multiples of $\underline{z}$ of $\{F=1\}$, and, $y \in$ Interior $F^{-1}([0,1])$. The set $\underline{z}-\Gamma$ and three level sets of $F$ are sketched in the Figure 5 .

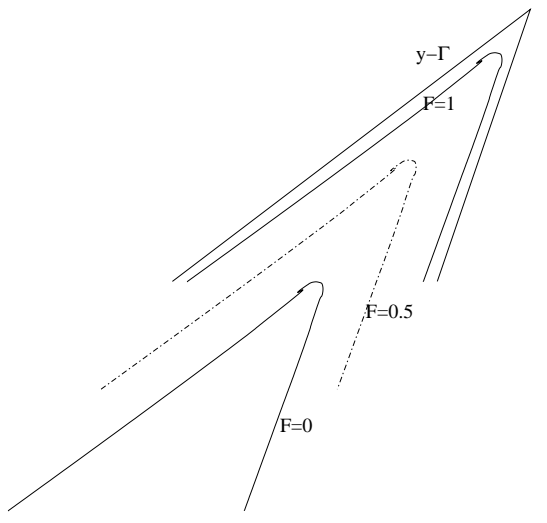

FiG. 5 
This is achieved by setting

$$
F:=A\left(t-g^{\varepsilon}(x-\underline{x} t)\right)+1 .
$$

Let $M:=\max _{x \in \omega} g^{\varepsilon}(x)$, and choose $A:=-1 / M$. With this choice, the set $\{F=0\}$ has maximal element touching $\{t=0\}$ where the maximum of $g$ is attained.

We next show that the surfaces $\{F=\sigma\}$ with $0 \leq \sigma \leq 1$ are space like, verifying hypothesis iii of Proposition 5. Lemma 7 proves that $\left(1-\nabla_{\tilde{x}} g^{\varepsilon}\right) \in \tilde{\mathcal{T}}_{1}$. Thus the conormal variety to the level sets satisfy

$$
N^{*}\left(\left\{\tilde{t}=g^{\varepsilon}(\tilde{x})\right\}\right) \subset \tilde{\mathcal{T}} \cup-\tilde{\mathcal{T}},
$$

where $\tilde{\mathcal{T}}$ is the open dual cone to $\tilde{\Gamma}$.

After the shear $S$ from (5), this yields

$$
N^{*}\left(\left\{t=g^{\varepsilon}(x-\underline{x} t)\right\}\right) \subset \mathcal{T} \cup-\mathcal{T}
$$

where $\mathcal{T}$ is the open dual cone to $\Gamma$.

Since $\Gamma(y) \subset \Gamma$, it follows that $\mathcal{T}(y) \supset \mathcal{T}$. Therefore the conormals to $\{t=$ $\left.g^{\varepsilon}(x-\underline{x} t)\right\}$ belong to $\mathcal{T}(y) \cup-\mathcal{T}(y)$ since they belong to $\mathcal{T} \cup-\mathcal{T}$. Thus, the level surfaces of $F$ are space like.

Proposition 5 implies that $u$ vanishes in $\{0 \leq F \leq 1\} \subset \Omega$. Since $y \in$ Interior $F^{-1}([0,1])$ this completes the proof of the lemma.

Proof of Theorem 1. It suffices to show that $u$ vanishes in $\mathcal{E}_{\varepsilon}^{-}(y)$ as in Lemma 2.

By uniqueness in the Cauchy problem at the time like surface $\{t=0\}$, it follows that $u$ vanishes on a neighborhood in $[0, T] \times \mathbb{R}^{d}$ of the compact set $\mathcal{E}_{\varepsilon}^{-}(\underline{y}) \cap\{t=0\}$. In particular,

$$
\Sigma:=\left\{\sigma \in[0, \underline{t}]: u=0 \text { on } \mathcal{E}_{\varepsilon}^{-}(\underline{y}) \cap\{0 \leq t \leq \sigma\}\right\},
$$

contains a neighborhood of $\{\sigma=0\}$.

The set $\Sigma$ is a closed interval by definition. It suffices to show that $\Sigma=[0, \underline{t}]$. By connectedness, it suffices to show that $\Sigma$ is a relatively open subset of $[0, \underline{t}]$.

If not, $\Sigma=[0, \sigma]$ with $0<\sigma<\underline{t}$. In this case, there is a sequence of points $z_{n}:=\left(t_{n}, x_{n}\right) \in \mathcal{E}_{\varepsilon}^{-}(\underline{y})$ with

$$
\sigma<t_{n} \searrow \sigma, \quad u\left(z_{n}\right) \neq 0 .
$$

Passing to a subsequence we may suppose that $\left(t_{n}, x_{n}\right) \rightarrow(\sigma, x):=y \in \mathcal{E}_{\varepsilon}^{-}(\underline{y})$.

Apply Lemma 8 with the fixed cone $\Gamma:=\Gamma^{+, \varepsilon / 2}(y)$ and vertex point $z_{n}, \delta_{n}=$ $t_{n}-\sigma$.

Choose $n_{0}$ so that for $n>n_{0}$ and $p \in \Omega\left(z_{n}, \delta_{n}\right), \Gamma^{+}(p) \subset \Gamma^{+, \varepsilon / 2}(y) \subset \Gamma^{+, \varepsilon}(p)$. In particular, $\Omega\left(z_{n}, \delta_{n}\right) \subset \mathcal{E}_{\varepsilon}^{-}\left(z_{n}\right)$ Therefore, by definition of $\Sigma$ and $\sigma, u$ vanishes on $\Omega\left(z_{n}, \delta_{n}\right) \cap\left\{t=t_{n}-\delta_{n}=\sigma\right\}$ provided $n>n_{0}$.

Lemma 8 applies and proves that $u$ vanishes on $\Omega\left(z_{n}, \delta_{n}\right)$. In particular, $u\left(z_{n}\right)=0$ for $n>n_{0}$ which is contradicts (6). This contradiction implies that $\Sigma=[0, \underline{t}]$ and therefore that $u$ vanishes on $\mathcal{E}_{\varepsilon}^{-}(\underline{y})$. 
[1] M.D. Bronshtein, Smoothness of polynomials depending on parameters, Siberian Mat. Zh., 20 (1979), pp. 493-501 (Russian); English transl., Siberian Math. J., 20 (1980), pp. 347-352.

[2] M.D. BRonshstein, The Cauchy problem for hyperbolic operators with characteristics of variable multiplicity, Trudy Mosk. Mat. O., 41 (1980), pp. 83-99; English transl., Trans. Moscow. Math. Soc., 1 (1982), pp. 87-103.

[3] R. Courant, Methods of Mathematical Physics vol II, Interscience Publ., 1962.

[4] K. O. Friedrichs, Symmetric hyperbolic linear differential equations, Comm. Pure Appl. Math., 7 (1954), pp. 517-550.

[5] L. GÅRDING, Linear hyperbolic partial differential operators with constant coefficients, Acta Math., 85 (1951), pp. 1-62.

[6] L. HöRmANDER, Uniqueness theorems and estimates for normally hyperbolic partial differential equations of the second order, Tolfte Skandinaviska Matematikerkongressen, Lunds Universitets Matematiska Institution, Lund 1954, pp. 105-115.

[7] V. IvrII, Linear Hyperbolic Equations in Encyclopaedia of Mathematical Sciences vol. 33, Partial Differential Equations IV, eds. Yu. V. Egorov and M.A. Shubin, Springer 1993, Russiian original, Itogi nauki i tekhniki, Sovremennye problemy matematiki, Fundamental'nye napravleniya, vol 33, Differentsial'nye uravneniya s chastnymi proizvodnymi 4, Viniti, Moscow 1988.

[8] F. JoHn, On linear partial differential equations with analytic coefficients, Unique continuation of data, C.P.A.M., 2 (1949), pp. 209-253.

[9] J.L. Joly, G. MÉtivier, J. RaUCH, Hyperbolic domains of determinacy and Hamilton-Jacobi equatiions, J. Hyp. Part. Diff. Eq., to appear.

[10] P. LAx, Lectures on Hyperbolic Partial Differential Equations, Stanford Lecture Notes, 1963.

[11] J. Leray, Hyperbolic Differential Equations, Institute for Advanced Study, 1953.

[12] A. Marchaud, Sur les champs continus de demi cônes convexes et leurs intégrales, Compositio Math., 2 (1936), pp. 89-127.

[13] J. Rauch, Partial Differential Equations, Graduate Texts in Mathematics \#128, SpringerVerlag, 1991.

[14] S. Spagnolo and G. Taglialatela, Quasisymmetrizers and semilinear hyperbolic systems, in preparation.

[15] S. Wakabayashi, Remarks on hyperbolic polynomials, Tsukuba J. Math., 10 (1986), pp. 17-28. 
